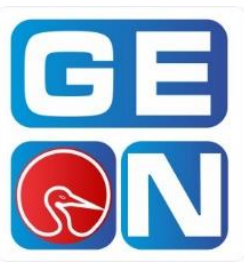

Revista GEON (Gestión, Organizaciones y Negocios.) ISSN: 2346-3910 en línea

revistageon@unillanos.edu.co

Universidad de los Llanos

Colombia

Almánzar Fortuna, Ricardo Joeli; Flores, Claudia Esmeralda.

Planificación estratégica en las empresas públicas de República Dominicana, caso

CDEEE

Revista GEON, Vol. 5, No. 2, 2018

Pág. 106-114

Disponible en: https://doi.org/10.22579/23463910.75

${ }^{\mathrm{i}}$ https://orcid.org/0000-0003-2353-397X

Esta publicación se encuentra bajo licencia: Creative Commons

ReconocimientoNoComercialSinObraDerivada 4.0 Internacional y la estrategia es uno de los factores principales de éxito o fracaso para un plan

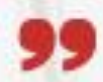

\title{
RevistaGEON
}

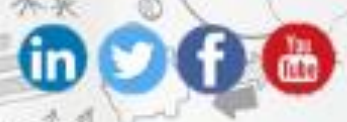




\section{Planificación estratégica en las empresas públicas de República Dominicana, caso CDEEE}

\section{Strategic Planning in Public Enterprises of Dominican Republic, case CDEEE}

Ricardo Joel Almánzar F

rj.almanzarf@gmail.com

Dr. Ciencias Económicas y Administrativas, Ucimexico, México

\section{Claudia Esmeralda Flores M}

claudiaefloresm@gmail.com

Dra. Ciencias Económicas y Administrativas, Ucimexico, México

Revista GEON Vol. 5 No 2 julio-diciembre 2018 


\title{
Resumen
}

La planificación de las empresas del Estado implica una serie de lineamientos estratégicos que deberían conciliar las buenas prácticas administrativas, así como el aseguramiento de la calidad de los procesos utilizados para garantizar que el uso de los recursos públicos se emplea de la manera correcta. La problemática en el sector eléctrico de la República Dominicana ha obligado al gobierno a plantearse un esquema que le permita dar una solución permanente a las dificultades de generación, compra y distribución de la energía, así como el mejoramiento de la estructura de servicios. Para ello se implementó en el 2013 un nuevo modelo de planificación estratégica basada en ejes estructurales, que son objeto de análisis en el presente artículo.

Palabras Clave: planificación, estrategia, energía, electricidad, recursos, procesos

\begin{abstract}
The planning of companies of the State involves a series of strategic guidelines that should reconcile the good administrative practices, as well as the assurance of the quality of the processes used to ensure that the use of public resources are used in the right way. The problem in the electricity sector in the Dominican Republic has forced the government to consider a scheme that allows you to provide a permanent solution to the difficulties of generation, purchase and distribution of energy, as well as the improvement of the service structure. For this was implemented in 2013 a new strategic planning model based on structural axes, which are analyzed in this article.
\end{abstract}

Keywords: Planning, Strategy, energy, electricity, resources, processes

\section{Introducción}

En todo acto de gestión pública o privada, la planificación es una tarea esencial que exige a quienes la elaboran, responder a preguntas esenciales sobre su gestión: ¿Quiénes somos? ¿Para quién trabajamos? ¿Qué hacemos? ¿Qué queremos ser y hacer a futuro? Sin embargo, este es solamente el inicio del proceso.

A lo largo de la historia, el uso de estrategias ha permitido diferenciar a 
individuos, grupos, líderes y civilizaciones enteras en cuestión del éxito o fracaso. Todos ellos pudieran tener objetivos similares como: sobrevivir, establecer imperios, ganar batallas, vencer a los oponentes, hacer sueños realidad, etc. La clave del éxito o fracaso se encuentra ligada al proceso de la planificación estratégica, aunque para cada caso la explicación se ubica en diferentes fases de la planeación.

En el presente artículo se revisa el caso de la Corporación Dominicana de Empresas Eléctricas Estatales (CDEEE) en su segundo ejercicio de planificación, según lo dispuesto en la Ley No. 498-06 en la República Dominicana, de la que surge el Sistema Nacional de Planificación e Inversión Pública. Se analiza, por lo tanto, el procedimiento que ha seguido la CDEEE para su planificación estratégica, contenido en el Plan Estratégico de la CDEEE para el periodo 2013-2016, a raíz de una serie de deficiencias que originaron problemas sistemáticos que por más de cinco décadas han quedado sin resolver; dando como origen el Plan Estratégico de la CDEEE, pautado para el período 20132106.

Las empresas públicas en República Dominicana han sido por tradición deficitarias, tanto en el aspecto económico como en el de la prestación de sus servicios y la estructura operativa de las mismas. Es a partir del primer gobierno del Doctor Leonel Fernández en el 1996 cuando adquieren cierto protagonismo y se inicia el cambio del modelo administrativo.
Si bien es cierto que se ha logrado algún avance con la inclusión de la carrera administrativa y además en la política de sueldos, cabe mencionar que el mayor obstáculo sigue siendo de voluntad política, lo que ha impedido una verdadera gestión pulcra y unos criterios de uniformidad en las instituciones del Estado.

La Corporación de Empresas Eléctricas Estatales, CDEEE, es una dependencia directa del gobierno dominicano, perteneciente al Ministerio de Energía y Minas, MEM, la que agrupa las distintas empresas generadoras en un conglomerado productivo. Su papel como regulador y transferencia de ingresos al Estado es de vital importancia para el desenvolvimiento

$\mathrm{y}$

ejecución presupuestal anual.

\section{metodológico \\ (i) Referente teórico $y$}

La metodología utilizada se basa en el análisis documental, en tanto que se recurre a recopilar datos plasmados en los planes estratégicos de la institución en cuestión, así también utilizando los esquemas de planificación estratégica dispuestos en los distintos recursos académicos facilitados para el análisis de caso.

La estrategia es el patrón de decisiones de la empresa que determina y revela sus objetivos, propósitos o metas, produce las principales políticas y planes para lograr esos objetivos y define el rango de negocio la empresa está persiguiendo, el tipo de organización económica y humana es o pretende ser, y la naturaleza 
de los derechos económicos y no económicos que se propone hacer contribuciones a sus accionistas, empleados, clientes y comunidades. (Andrew, 1971)

Por otra parte, como bien menciona Contreras (2013) "la estrategia de una empresa puede ser mejor que la de otra sin importar el tamaño, no necesariamente tiene que ser la más grande para poder salir adelante. Lo importante es que se tengan definidos los objetivos a los cuales se apunta, se cuente con el conocimiento y los deseos de echar a andar el proyecto hacia adelante" (sp.). Para esto se requiere primeramente la revisión o definición de la Misión, Visión y Valores, considerados como la fase filosófica o ideológica del proceso de planificación estratégica.

Luego viene la fase analítica, que consiste en realizar una revisión de los principales factores internos y externos, identificando tanto las oportunidades para el posicionamiento de la empresa, así como todos los factores que pudieran afectar su éxito, ya sean amenazas externas o las propias debilidades, además de las fortalezas (incluyendo recursos materiales y humanos). Una vez analizados estos factores, se puede proceder al establecimiento de objetivos (fase programática) a partir de las oportunidades o problemas identificados, siendo estos objetivos una propuesta para dar solución a los problemas o retos encontrados. A continuación, en la fase operativa se definirán las políticas de acción y estrategias detalladas que lleven al logro de los objetivos. Finalmente se trabaja una fase cuantitativa en la que se identifican los indicadores a utilizar, así como los valores esperados.

En tal sentido, siguiendo la línea de pensamiento de los autores, la estrategia como parte de un plan elaborado que busca lograr metas y alcanzar objetivos es aplicable a cualquier tipo de empresa, incluso al ser humano como individuo.

Unas 11 estrategias o enfoques para la planeación estratégica son las descritas por David (2003), según el propósito principal de la empresa para un determinado periodo de planeación. Las estrategias de integración vertical, por ejemplo, permiten a una empresa el control de distribuidores, proveedores y competidores (integración hacia delante, integración hacia atrás, integración horizontal); las estrategias de penetración en el mercado y desarrollo en el mercado, tienen que ver con la búsqueda del aumento de la participación o introducción de sus productos o servicios a través de la mercadotecnia; la diversificación concéntrica y horizontal, tienen que ver con la adición de productos o servicios ya sea relacionados o no con los clientes actuales; el desarrollo de productos es la búsqueda de incrementos de ventas mejorando o desarrollando productos $\mathrm{y}$ servicios; la enajenación consiste en la venta de una división o parte de la empresa; el recorte de gastos implica la disminución de adquisiciones y costos; la liquidación consiste en la venta de activos de la empresa en partes.

Como explica Vetter (2012), sin embargo, la razón por la que una de cada 10 estrategias pueda fracasar, adjudicándose a una "pobre ejecución" y 
cita Charan y Bossidy (2002) que definen como los 3 elementos de la ejecución. El primer elemento es en realidad una lista de actitudes de liderazgo: cercanía con el equipo, realismo, objetivos y prioridades, compromiso, sistema de recompensas, expansión de capacidades del equipo y conocimiento propio.

El segundo elemento se refiere a la cultura de Ejecución en la organización, de la cual el líder es el responsable al asegurar que los empleados entiendan lo que se espera de ellos y cómo lograrlo, así como aplicar el sistema de recompensas. El tercer elemento consistirá en tener a las personas indicadas en los lugares correctos. Así, se espera que una empresa exitosa en la ejecución de sus estrategias sea aquella en la que todos conocen sus responsabilidades, además del impacto de sus acciones y no se cuestionen las decisiones una vez tomadas, sin embargo, se incluyan todos los niveles gerenciales para la toma de decisiones; los gerentes operativos deberán conocer los resultados de las evaluaciones.

\section{(ii) Resultados}

Formulación del plan. En cuanto a su metodología, la Corporación Dominicana de Empresas Eléctricas Estatales (CDEEE) fundamentó la formulación de su Plan 2013-2016 en la cadena de valor, donde ubica su acción para lograr el impacto final deseado partiendo desde la definición de recursos, los cuales a su vez se usan para generar productos y a partir de estos se logran resultados que finalmente llevan a un impacto. En este caso, las operaciones son el punto intermedio entre los recursos y productos, donde la CDEEE se asume como líder para el cumplimiento de las metas definidas, tanto para los productos intermedios y finales. Aquí se resalta que es esta metodología la que se recomienda por el Ministerio de Economía, Planificación y Desarrollo (MEPyD) para la documentación de planes estratégicos de instituciones públicas.

Antes de abordar la primera fase del plan, la CDEEE resume los datos relevantes de su ejercicio anterior, incluyendo un cuadro de indicadores con sus respectivas metas y resultados obtenidos al finalizar dicho periodo (20102012). De este primer ejercicio se rescata un acercamiento del $80 \%$ de precisión entre las metas definidas y sus resultados finales para la mayor parte de los indicadores, aunque la mayoría de las metas (en sentido estricto) no se cumplen, dejando un mayor reto principalmente para el rubro de pérdidas de energía, de las que se esperaba alcanzar un $22 \%$ pero se obtuvo $35 \%$.

En cuanto a la fase ideológica o filosófica de la planeación estratégica propuesta por Medianero (s.f., p.7), la CDEEE responde al "¿Quiénes somos?" definiéndose como una "empresa autónoma de servicio público, con patrimonio propio y personalidad jurídica" (CDEEE, p. 16) a cargo de liderar y coordinar a las empresas eléctricas estatales que distribuyen y comercializan electricidad en las diferentes zonas de la República Dominicana (EDENORTE, EDESUR, EDEESTE), además de la Unidad de Electrificación Rural y Suburbana (UERS) encargada de electrificar 
comunidades rurales y sub-urbanas con fuentes renovables y no renovables, así como fomentar la cultura del ahorro y manejo responsable de la energía. Se encuentran también a su cargo la Empresa de Transmisión Eléctrica Dominicana (ETED) encargada de las redes de alta tensión y las subestaciones de transmisión del Sistema Eléctrico Nacional Interconectado (SENI); y la Empresa de Generación Hidroeléctrica Dominicana (EGEHID) ocupada de la generación y administración de energía hidroeléctrica a partir de aguas en movimiento como ríos, mareas, saltos de agua, etc. Como parte de su identidad, la CDEEE reconoce en este mismo apartado su relación con otras instituciones del sector eléctrico: (CNE)

Comisión Nacional de Energía

Superintendencia de Electricidad de la República Dominicana (SIE)

Organismo Coordinador del Sistema Eléctrico Nacional Interconectado (OC)

Misión:

"Liderar la gestión de las empresas eléctricas estatales, para garantizar un servicio de electricidad continuo y eficiente";

Visión:

"Lograr un sector eléctrico auto sostenible que contribuya al desarrollo del país";

\section{Valores:}

Integridad: conducta honesta, recta y respetuosa hacia las personas y la institución.
Compromiso: en los objetivos institucionales que impactan a la sociedad y el medio ambiente.

Calidad: mejora permanente en todos los servicios con un equipo que interactúa en confianza y seguridad

Trabajo en equipo: esfuerzos aunados para el alcance de los objetivos comunes, fortaleciendo el espíritu colectivista

Perseverancia: firmeza y constancia en la consecución de los propósitos

En la fase de análisis se describe el principal problema a nivel nacional con el suministro eléctrico, el cual impacta negativamente al desarrollo de la economía: el balance comercial deficitario provocado por un esquema tarifario por debajo del costo de producción del cual el gobierno asume el déficit, por lo que se dificulta el abastecimiento de la demanda de manera constante. Este problema central se conforma por: altos precios de compra de energía, pérdidas técnicas y no técnicas de energía, e inversiones no focalizadas ni sistemáticas en el sector de transmisión y distribución.

Así, mientras las empresas distribuidoras deben pagar 2 mil millones de dólares por la compra de energía a las empresas generadoras, los ingresos por venta de energía no pasan de 1,500 millones de dólares. Las pérdidas calculadas se componen combinando este déficit con el hurto y uso no racional de la energía, superando en conjunto los mil millones de dólares. A partir de las tablas de balance y de evolución de los indicadores comerciales de las empresas dominicanas de energía eléctrica, la CDEEE identifica cinco retos: 
Puesta en marcha de acciones y proyectos

Financiamientos públicos y privados

Participación de todos los sectores y población en general para la solución integral

Documentación de los resultados al final del periodo de gestión, rescatando las lecciones aprendidas en la puesta en marcha

Modelo de mercado mayorista

Para la fase programática se definen 3 ejes estratégicos referentes a: 1) Modificación de la matriz existente y ampliación del parque existente, 2) reducción de pérdidas y 3) eficiencia en la gestión. De estos se desprenden los siguientes objetivos generales $\mathrm{y}$ específicos:

1. Objetivo general: Asegurar un suministro confiable de electricidad a precios competitivos

Incrementar 1,500 megavatios al parque de generación

Mejorar los precios de compra de energía y reducir costos de generación

Reducir el despacho de unidades de altos costos e ineficientes

2. Objetivo general: Mejorar la infraestructura de transmisión y distribución de electricidad, que opere con estándares de calidad y confiabilidad del servicio establecido por las normas.

Reducir 10.4 PP las pérdidas técnicas y comerciales al 2016.

3. Objetivo general: Mejorar la gestión de las empresas eléctricas estatales.
Aumentar la satisfacción del cliente.

Disminuir las pérdidas comerciales administrativas.

Mejorar el flujo de caja de las empresas.

Optimizar los recursos tecnológicos para mejorar la calidad del servicio.

Adecuar la normativa existente a las necesidades de las empresas y los clientes.

\section{Implementación del plan}

La etapa de implementación está contenida en varias matrices en las que se despliega cada uno de los objetivos y líneas de acción específicas en distintas perspectivas, las cuales son comercial, financiera, tecnología y sistemas, y fortalecimiento institucional.

También se prevé la revisión de los indicadores a partir de reuniones periódicas para dar seguimiento al proceso de ejecución, aunque no se determinan por completo los todos los aspectos a evaluar.

Un punto importante es que no existe un cronograma general de cada una de las acciones a realizarse, así como los proyectos contemplados para cada uno de los ejes estratégicos definidos en la etapa de planeación.

Es notable el hecho de que a pesar de que se ha pensado un plan estratégico, no se evidencia una integración del personal, es decir, ¿Cuál es el papel de la gente de la empresa en la implementación de la estrategia? Según lo expuesto por Medianero, Vetter y otros autores, debe existir una relación directa con la implementación de la estrategia y el rol del 
recurso humano de la empresa, esto con la finalidad de dar a conocer lo que quiere la alta gerencia, cómo lo quiere, cuándo y dónde lo quiere, así como también los medios que se utilizará para lograrlo.

Un aspecto interesante es el uso de ejes estratégicos, mencionado anteriormente, lo cual obedece al uso del cuadro de mando integral, que, aunque no está explícitamente nombrado, sí forma parte de la planificación, esto es al proponer la perspectiva financiera, clientes, operaciones y procesos y aprendizaje.

El problema está específicamente en que se presentan como aisladas estas perspectivas, cuando deben estar íntimamente compenetradas para su mejor manejo y, en tanto que se trabaja con una serie de indicadores conviene tenerlos agrupados y categorizados, cada uno atado a sus factores de éxito (Miranda, 2007). Éste indica además que un enlace adecuado entre los distintos indicadores se logra a partir del mapa estratégico, el cual se usa para interconectar cada objetivo en una secuencia lógica que va desde una perspectiva a otra, con lo que se prioriza ideológicamente cuáles deben cumplirse primero para poder pasar hacia adelante.

\section{(iii) Discusión y conclusiones}

Una primera impresión al revisar este Plan Estratégico de la CDEEE es el acatamiento a lo que el Ministerio de Economía, Planificación y Desarrollo (MEPyD) establece desde la metodología de formulación, así como una plena consideración, para sus contenidos, del Plan de Gobierno de 2012-2016 elaborado por el Presidente de la República Dominicana, Lic. Danilo Medina Sánchez, donde se enfatiza la integración y articulación sectorial de la economía para el desarrollo del país.

Se distinguen entre sus ejes de acción estrategias enfocadas al recorte de gastos tanto en términos de gestión administrativa, costes por generación y compra de energía, a partir de diferentes proyectos. Por otra parte, se plantean estrategias de desarrollo de productos al procurar mejoras en la calidad de los servicios, modificaciones en cuanto a la potencia de energía ofrecida y la introducción de fuentes alternativas para una transición del uso de combustibles derivados del petróleo al uso de fuentes de energía renovables.

Sin embargo, a pesar de que se ha logrado un avance con el plan estratégico, la empresa no ha dado el salto esperado, pues si bien es cierto que en cuestiones de generación se ha avanzado, no es menos cierto que aún existen muchos hogares sin acceso a la energía eléctrica, lo cual implica un fallo en el logro de los objetivos planteados.

Algo para destacar es que la matriz energética continúa bajo la misma composición, datos que pueden verificarse en el portal de transparencia de la empresa, $\mathrm{y}$, donde se evidencia que la producción de energía en más del $50 \%$ aún depende de los combustibles, por lo tanto, un objetivo no alcanzado por el plan, el cual finaliza el próximo 16 de agosto del presente año 2016.

Aunque la CDEEE ha considerado para su plan, los posibles riesgos para el 
logro de los objetivos planteados, no se detectan políticas de acción claras para garantizar que estas estrategias puedan llevarse a cabo, tampoco se menciona un sistema de recompensas que al estar claramente definido coadyuve en las mejoras de calidad de servicio y mejore la cultura de ejecución.

Muchas de estas estrategias tienen un alto impacto para el logro de los objetivos, y dependen de factores externos sobre los que no se tiene garantía, tales como las decisiones de gobierno o la asignación puntual de los recursos esperados, en cuyo caso la acción a tomar consiste en "asumir". Un ejemplo de esto es la Ley de Energías Renovables en 2008, que concedería exenciones de impuestos para las iniciativas de producción de energía renovable, sin embargo, una reforma fiscal en 2012 eliminó estos incentivos de exenciones fiscales mayores.

Un aspecto que sí pudiera estar bajo el control de la CDEEE es la planificación financiera más rigurosa y un mayor control de medición de los indicadores relacionados con el desempeño de los empleados.

La conexión entre la parte financiera y la estrategia es uno de los factores principales de éxito o fracaso para un plan, según la documentación revisada. Por otra parte, el desempeño de los empleados debe proyectar su completo compromiso, y las revisiones en el seguimiento deben aportar datos que indiquen su avance, de lo contrario, será necesario integrar una estrategia de recorte y sustitución del personal para favorecer el cumplimiento cabal de las estrategias contenidas en el plan.

\section{Referencias}

Contreras R., 2013, El concepto de estrategia como fundamento de la planeación estratégica, Revista Pensamiento y Gestión, $N^{\circ}$ 35, Colombia

Corporación Dominicana de Empresas Eléctricas Estatales (2013) Plan Estratégico 20132016 CDEEE. Recuperado el 8 de junio de 2016, de: http://pactoelectrico.do/wpcontent/uploads/2015/11/01.-Plan-EstrategicoCDEEE-2013-2016.pdf

David, F., 2003, Conceptos de Administración Estratégica. México: Pearson, $9^{a}$ ed.

Medianero, D. (s.f.) Guía metodológica para el diseño de planes estratégicos en el sector público. Un enfoque participativo. Recuperado el 15 de junio de 2016, de: http://www.pnuma.org/aguamiaac/REGIONAL/ MATERIAL\%20ADICIONAL/BIBLIOGRAFI AWEBGRAFIA\%20(2)/Guia\%20metodologica $\% 20$ planes \%20estrategicos\%20sector\%20public o.pdf

Miranda R., 2007, Elaboración del Cuadro de Mando Integral, Universidad Nacional, Heredia, Costa Rica.

Vetter, G., 2012, La ejecución estratégica, o cómo hacer que las cosas ocurran. Center forInnovativeExecution (CFIE), Cuadernos de conocimiento, pp. 1-16. Recuperado el 22 de junio de 2016, de:http://www.eoi.es/sc/webeoi/documentos/Arti culo_EjecucionEstrategica.pdf 